\title{
SYNTHESIS AND BIOLOGICAL ACTIVITY OF SOME NEW 1,2,4- TRIAZINO[6,1-B]QUINAZOLINE DERIVATIVES.
}

BY

Mervat M. El-Enany, Afaf K. El-Ansary, Mona M. Abdel-Gawad and Ehab M.

Gedawy.

FROM

Pharmaceutical Organic Chemistry Department, Faculty of Pharmacy, Cairo University, Cairo 11562, Egypt.

\section{ABSTRACT}

In this study, novel 3-substituted 1,2,4-triazino[6,1-b]quinazolines 4a-e, 8substituted-1H-[1,2,4]triazino[6,1-b]quinazoline-2,4,10(3H)-triones $8 \mathrm{a} \& \mathrm{~b}$ and 2substituted 1,2,4-triazino[6,1-b]quinazolines 9a-d, $10 \& 11$ were synthesized from 3amino-6-bromo-2-ethoxycarbonylquinazolin-4(3H)-one (2b) and 3-amino-2aminocarbonylquinazolin-4(3H)-one $7 \mathrm{a} \& \mathrm{~b}$. Some of the newly synthesized compounds were tested for their antimicrobial activity and analgesic activity.

Depending on the obtained results, the newly synthesized compounds possess significant analgesic activity and mild antimicrobial activity.

\section{Introduction}

Quinazoline derivatives have been reported to possess diverse biological activities such as analgesic (Alafeefy et al., 2010), anti-inflammatory (Alagarsamy et al., 2007; Chandrika et al., 2008; Giri et al., 2009), antimicrobial (Minu et al., 2008; Panneerselvam et al., 2009; Ryu et al., 2012) and anticancer (Noolvi et al., 2011 and Mulakayala et al., 2012). In addition 1,2,4-triazine derivatives were found to possess antimicrobial activity (Taha M.A.M., 2007 and Elsilk et al., 2010), antitumor activity (Gucky' et al., 2010). Finally, condensed [1,2,4]triazinoquinazoline were reported to have antibacterial and antifungal activity (Abdel-Mageed et al., 1988; Abdel-Hamide et al., 1997; Abdel-Hamide, 2001; Ghorab et al. 2013), anti-inflammatory activity (Bansal et al., 2001 and Hussein, 2012), anticancer activity (Kovalenko et al. 2013) and analgesic activity (Pathak et al. 1995).

The above findings directed the attention to the synthesis of certain substituted $[1,2,4]$ triazino[6,1-b]quinazoline hoping that they may possess antimicrobial activity and analgesic activity.

\section{Discussion}

2-amino-5-bromobenzoic acid hydrazide (1b) was prepared from reacting methyl 5-bromoanthranilic acid with hydrazine hydrate adopting Casgranda's precautions (Casgrande et al. 1965). Meanwhile synthesis of 3-Amino-2ethoxycarbonylquinazolin-4(3H)-one $(2 \mathrm{a})$ was prepared via heating 2-aminobenzoic acid hydrazide (1a) with excess diethyl oxalate according to the reported method (George et al. 1971). Finally, 3-Amino-2-aminocarbonylquinazolin-4(3H)-one (7a) was prepared via aminolysis of corresponding amino ester 2a adopting George's conditions (George et al. 1971). 
In this study, Certain 3-substituted 1,2,4-triazino[6,1-b]quinazolines 4a-g were synthesized from reacting 3-Amino-6-bromo-2-ethoxycarbonylquinazolin-4(3H)-one (2b) with triethyl orthoformate to afford 6-Bromo-2-ethoxycarbonyl-3-ethoxymethylenaminoquinazolin-4(3H)-one (3). The latter was cyclized with different aliphatic or aromatic amines to afford $4 \mathrm{a}-\mathrm{g}$. Meanwhile, reaction of $2 \mathrm{~b}$ with phenyl isocyanate and formamide yielded $5 \& 6$ respectively. In addition, 8-Substituted-1H$[1,2,4]$ triazino[6,1-b]quinazoline-2,4,10(3H)-triones $8 \mathrm{a} \& \mathrm{~b}$ were prepared via fusion 3 amino-2-aminocarbonylquinazolin- $4(3 \mathrm{H})$-one $7 \mathrm{a} \& \mathrm{~b}$ with urea. Finally, 2-substituted 1,2,4-triazino[6,1-b]quinazolines $9 \mathrm{a}-\mathrm{d}, 10 \& 11$ from reacting $7 \mathrm{a} \& \mathrm{~b}$ with different substituted aromatic aldehydes, chloroacetyl chloride or trifluoroacetic anhydride.

\section{Experimental}

Melting points were determined on a Griffin apparatus and are uncorrected.

Microanalysis for $\mathrm{C}, \mathrm{H}$ and $\mathrm{N}$ were carried out at Microanalytical Centre, Cairo University and Organic Microanalyses Section, National Research Centre. IR spectra were recorded on Schimadzu 435 spectrometer using $\mathrm{KBr}$ discs. ${ }^{1} \mathrm{H}$ NMR spectra were performed on a Varian Gemini $200 \mathrm{MHz}$, Microanalytical Centre, Cairo University using TMS as an internal standard and chemical shift values are recorded in ppm on $\delta$ scale. Mass spectra were run at 70 ev on HP-5988A Mass spectrometer. Progress of the reactions was monitored by TLC using aluminium sheets percoated with UV fluorescent silica gel (Merck 60 F254) and were visualized using UV lamp and iodine vapour. The used solvent system was chloroform : benzene : methanol [6: $3.5: 0.5]$.

\section{3-Amino-6-bromo-2-ethoxycarbonylquinazolin-4(3H)-one (2b).}

A mixture of 2-amino-5-bromobezoic acid hydrazide $1 \mathrm{~b}(15.18 \mathrm{~g}, 0.066 \mathrm{~mol})$ in diethyl oxalate $(19.5 \mathrm{~mL})$ was heated under reflux with stirring in an oil bath at $180{ }^{\circ} \mathrm{C}$ for $6 \mathrm{~h}$. The excess diethyl oxalate was distilled off under vacuum to give a semisolid mass which upon treatment with ethanol afforded white crystalline soild \& finally crystalized from DMF.

Yield: $60.0 \%$; mp: $165-167^{\circ} \mathrm{C}$; IR( $\left(\mathrm{cm}^{-1}\right) ; 3330,3270(\mathrm{NH}), 2975$ (C-H aliphatic), 1738, $1680(2 \mathrm{C}=\mathrm{O}) ;{ }^{1} \mathrm{H}$ NMR (DMSO-d $): 1.4\left(\mathrm{t}, 3 \mathrm{H}, \mathrm{CH}_{3}\right), 4.4\left(\mathrm{q}, 2 \mathrm{H}, \mathrm{CH}_{2}\right), 5.95$ (br. S., $2 \mathrm{H}, \mathrm{NH}_{2}, \mathrm{D}_{2} \mathrm{O}$ exchangeable), 7.6-8.3 (m, 3H, Ar-H); MS: m/z $314\left(\mathrm{M}+3^{+}, 6.86 \%\right)$, $313\left(\mathrm{M}+2^{+}, 38.82 \%\right), 312\left(\mathrm{M}+1^{+}, 7.27 \%\right), 311\left(\mathrm{M}^{+}, 44.18 \%\right), 74.95(100 \%)$; Anal. for $\mathrm{C}_{11} \mathrm{H}_{10} \mathrm{BrN}_{3} \mathrm{O}_{3}$ Calcd. (Found): C, 42.32(42.35); H, 3.23(3.69); N, 13.46(13.72).

\section{6-Bromo-2-ethoxycarbonyl-3-ethoxymethylenaminoquinazolin-4(3H)-one (3)}

A solution of 3-amino-6-bromo-2-ethoxycarbonylquinazolin-4 $(3 \mathrm{H})$-one $(2 \mathrm{~b})$ $(3.12 \mathrm{~g}, 0.01 \mathrm{~mol})$ in triethyl orthoformate $(20 \mathrm{~mL})$ was heated under reflux for $1.5 \mathrm{~h}$. The excess triethyl orthoformate as well as the formed ethanol were evaporated under reduced pressure. The residue was crystallized from petroleum ether to afford solid.

Yield: 84.0\%; mp: 80-82 ${ }^{\circ} \mathrm{C}$; IR( $\left(\mathrm{cm}^{-1}\right) ; 2960$ (C-H aliphatic), 1740, $1680(2$ $\mathrm{C}=\mathrm{O}) ;{ }^{1} \mathrm{H}$ NMR (DMSO-d $)$ ) $1.4\left(\mathrm{t}, 6 \mathrm{H}, 2 \mathrm{CH}_{3}\right), 4.3-4.4\left(\mathrm{q}, 2 \mathrm{H}, \mathrm{CH}_{2}\right), 4.41-4.5(\mathrm{q}, 2 \mathrm{H}$, $\left.\mathrm{CH}_{2}\right)$, 7.2-7.8 (m, 3H, Ar-H), $8.6(\mathrm{~s}, 1 \mathrm{H},-\mathrm{CH}=\mathrm{N})$; Anal. for $\mathrm{C}_{14} \mathrm{H}_{14} \mathrm{BrN}_{3} \mathrm{O}_{4}$ Calcd. (Found): C, 45.67(46.20); H, 3.85(4.37); N, 11.41(11.48). 


\section{General procedure for the synthesis of 4a-e:}

To a solution of 6-Bromo-2-ethoxycarbonyl-3-ethoxymethyl-enaminoquinazolin $-4(3 \mathrm{H})$-one $(3)(4.05 \mathrm{~g}, 0.011 \mathrm{~mol})$ in toluene $(50 \mathrm{~mL})$, the appropriate amine $(0.01 \mathrm{~mol})$ was added. The reaction mixture was heated on water bath at $50{ }^{\circ} \mathrm{C}$ for $10 \mathrm{~min}$. (in case of aliphatic amines), or heated under reflux for $1.5 \mathrm{~h}$. (in case of aromatic amines). The reaction mixture was then cooled, filtered off. The produced solid was washed with chloroform then crystallized from the appropriate solvent to afford 4a-e.

\section{8-Bromo-3-(4-methoxyphenyl)-3H-[1,2,4]triazino[6,1-b]quinazoline-4,10-dione (4a)}

Yield: $73.0 \%$ (acetic acid); mp: $>300{ }^{\circ} \mathrm{C}$; IR $\left(\mathrm{cm}^{-1}\right) ; 2970$ (C-H aliphatic), 1720, $1680(2 \mathrm{C}=\mathrm{O}) ; \mathrm{MS}: \mathrm{m} / \mathrm{z} 401\left(\mathrm{M}+3^{+}, 19.50 \%\right), 400\left(\mathrm{M}+2^{+}, 100 \%\right), 399\left(\mathrm{M}+1^{+}, 23.17 \%\right)$, $398\left(\mathrm{M}^{+}\right.$, 95.50\%); Anal. for $\mathrm{C}_{17} \mathrm{H}_{11} \mathrm{BrN}_{4} \mathrm{O}_{3}$ Calcd. (Found): C, 51.14(50.91); $\mathrm{H}$, 2.77(2.50); N, 14.03(14.22).

\section{8-Bromo-3-methyl-3H-[1,2,4]triazino[6,1-b]quinazoline-4,10-dione (4b)}

Yield: $70.0 \%$ (acetic acid); mp: $>300{ }^{\circ} \mathrm{C}$; IR $\left(\mathrm{cm}^{-1}\right) ; 2970$ (C-H aliphatic), 1710, 1670 (2 C=O); Anal. for $\mathrm{C}_{11} \mathrm{H}_{7} \mathrm{BrN}_{4} \mathrm{O}_{2}$ Calcd. (Found): C, 43.02(42.80); H, 2.29(2.10); $\mathrm{N}, 18.24(18.14)$.

\section{8-Bromo-3-isopropyl-3H-[1,2,4]triazino[6,1-b]quinazoline-4,10-dione (4c)}

Yield: $95.0 \%$ (toulene); mp: > $300{ }^{\circ} \mathrm{C}$; IR $\left(\mathrm{cm}^{-1}\right) ; 2970$ (C-H aliphatic), 1710, $1650(2 \mathrm{C}=\mathrm{O})$; ${ }^{1} \mathrm{H}$ NMR (DMSO-d $\left.\mathrm{d}_{6}\right): 0.93\left(\mathrm{~d}, 6 \mathrm{H}, 2 \mathrm{CH}_{3}\right), 2.07-2.11(\mathrm{~m}, 1 \mathrm{H}, \mathrm{CH}), 7.8-$ 8.3 (m, 4H, Ar-H + -N=CH); Anal. for $\mathrm{C}_{13} \mathrm{H}_{11} \mathrm{BrN}_{4} \mathrm{O}_{2}$ Calcd. (Found): $\mathrm{C}$, 46.56(46.21); $\mathrm{H}, 3.28(3.29)$; $\mathrm{N}, 16.71(16.69)$.

\section{8-Bromo-3-isobutyl-3H-[1,2,4]triazino[6,1-b]quinazoline -4,10-dione (4d)}

Yield: $95.0 \%$ (toulene); mp: > $300{ }^{\circ} \mathrm{C}$; IR $\left(\mathrm{cm}^{-1}\right)$; 2970 (C-H aliphatic), 1720, $1650(2 \mathrm{C}=\mathrm{O})$; MS: m/z $351\left(\mathrm{M}+3^{+}, 13.35 \%\right), 350\left(\mathrm{M}+2^{+}, 78.2 \%\right), 349\left(\mathrm{M}+1^{+}, 15.93\right.$ $\%), 348\left(\mathrm{M}^{+}, 77.20 \%\right), 294.90(100 \%)$; Anal. for $\mathrm{C}_{14} \mathrm{H}_{13} \mathrm{BrN}_{4} \mathrm{O}_{2}$ Calcd. (Found): $\mathrm{C}$, 48.13(48.68); H, 3.72(3.84); N, 16.04(16.05).

\section{8-Bromo-3-cyclohexyl-3H-[1,2,4]triazino[6,1-b]quinazoline-4,10-dione (4e)}

Yield: $95.0 \%\left(\mathrm{CHCl}_{4} / \mathrm{methanol}\right)$; $\mathrm{mp}:>300{ }^{\circ} \mathrm{C}$; IR $\left(\mathrm{cm}^{-1}\right) ; 2970(\mathrm{C}-\mathrm{H}$ aliphatic), 1710, $1660(2 \mathrm{C}=\mathrm{O}) ;{ }^{1} \mathrm{H}$ NMR (DMSO-d $\left.)_{6}\right): 1.20-1.88\left(\mathrm{~m}, 10 \mathrm{H}, \mathrm{C}-\mathrm{H}\right.$ of $\left.5 \mathrm{CH}_{2}\right), 4.45(\mathrm{~m}$, $1 \mathrm{H}, \mathrm{CH}$ cyclohexyl), 7.80-8.11 (m, 4H, Ar-H + -N=CH); Anal. for $\mathrm{C}_{16} \mathrm{H}_{15} \mathrm{BrN}_{4} \mathrm{O}_{2}$ Calcd. (Found): C, 51.21(51.80); H, 4.02(4.41); N, 14.93(14.77).

methyl 2-(8-bromo-4,10-dioxo-4,10-dihydro-3H-[1,2,4]triazino[6,1-b] quinazolin-3yl)benzoate (4f)

Yield: $75.0 \%$ (pet. ether); mp: $180-182{ }^{\circ} \mathrm{C}$; IR $\left(\mathrm{cm}^{-1}\right) ; 2970$ (C-H aliphatic), 1700, $1660(2 \mathrm{C}=\mathrm{O})$; MS: $\mathrm{m} / \mathrm{z} 429\left(\mathrm{M}+3^{+}, 13.41 \%\right), 428\left(\mathrm{M}+2^{+}, 56.26 \%\right), 427\left(\mathrm{M}+1^{+}\right.$, $14.44 \%), 426\left(\mathrm{M}^{+}, 56.26 \%\right), 369(100 \%)$; Anal. for $\mathrm{C}_{18} \mathrm{H}_{11} \mathrm{BrN}_{4} \mathrm{O}_{4}$ Calcd. (Found): $\mathrm{C}$, 50.60(50.54); H, 2.59(2.45); N, 13.11(13.08).

\section{3-Amino-8-Bromo-3H-[1,2,4]triazino[6,1-b]quinazoline-4,10-dione (4g)}

Yield: $70.0 \%$ (acetic acid); mp: 296-298 ${ }^{\circ} \mathrm{C}$; IR $\left(\mathrm{cm}^{-1}\right)$; 3210-3200 $\left(\mathrm{NH}_{2}\right), 2970$ (C-H aliphatic), 1710, $1670(2 \mathrm{C}=\mathrm{O})$; Anal. for $\mathrm{C}_{10} \mathrm{H}_{6} \mathrm{BrN}_{5} \mathrm{O}_{2}$ Calcd. (Found): $\mathrm{C}$, 38.98(39.20); H, 1.96(2.20); N, 22.73(22.56). 


\section{General procedure for the synthesis of 5 a\&b:}

Phenyl iso(thio)cyanate $(0.006 \mathrm{~mol})$ was added to a solution of 6-Bromo-2ethoxycarbonyl-3-ethoxymethyl-enaminoquinazolin-4(3H)-one (3) (1.56g, $0.005 \mathrm{~mol})$ in glacial acetic acid $(15 \mathrm{~mL})$. The reaction mixture was heated under reflux for $6 \mathrm{~h}$., then allowed to cool. The separated yellow precipitate was filtered off and crystallized from acetic acid to yield $5 \mathrm{a} \& \mathrm{~b}$.

\section{8-Bromo-3-phenyl-1H-[1,2,4]triazino[6,1-b]quinazoline-2,4,10(3H)-trione (5a)}

Yield: 85.0\%; mp: > $300{ }^{\circ} \mathrm{C}$; IR $\left(\mathrm{cm}^{-1}\right) ; 3150(\mathrm{NH}), 1740,1680,1640(3 \mathrm{C}=\mathrm{O})$; MS: $\mathrm{m} / \mathrm{z} 386\left(\mathrm{M}+2^{+}, 26.47 \%\right), 384\left(\mathrm{M}^{+}, 25.52 \%\right), 119$ (100\%); Anal. for $\mathrm{C}_{16} \mathrm{H}_{9} \mathrm{BrN}_{4} \mathrm{O}_{3}$ Calcd. (Found): C, 49.89(49.58); H, 2.35(2.50); N, 14.54(14.50).

\section{8-Bromo-3-phenyl-2-thioxo-1H-[1,2,4]triazino[6,1-b]quinazoline-4,10(3H)-dione} (5b)

Yield: $80.0 \%$; mp: $>300{ }^{\circ} \mathrm{C}$; IR $\left(\mathrm{cm}^{-1}\right)$; $3250(\mathrm{NH}), 1680-1650$ (2 overlapped $\mathrm{C}=\mathrm{O}$ ); Anal. for $\mathrm{C}_{16} \mathrm{H}_{9} \mathrm{BrN}_{4} \mathrm{O}_{2} \mathrm{~S}$ Calcd. (Found): $\mathrm{C}, 47.90(47.85) ; \mathrm{H}, 2.26(2.64) ; \mathrm{N}$, 13.96(13.83).

\section{8-Bromo-3H-[1,2,4]triazino[6,1-b]quinazoline-4,10-dione (6)}

A solution of 6-Bromo-2-ethoxycarbonyl-3-ethoxymethyl-enaminoquinazolin$4(3 \mathrm{H})$-one $(3)(3.12 \mathrm{~g}, 0.01 \mathrm{~mol})$ in formamide was heated under reflux for $40-50 \mathrm{~min}$., then allowed to cool to room temperature. The separated white precipitate was collected by filteration and crystallized from acetic acid.

Yield: $65.0 \%$ (pet. ether); $\mathrm{mp}:>300{ }^{\circ} \mathrm{C}$; IR $\left(\mathrm{cm}^{-1}\right)$; 3300-3100 (NH), 1690-1680 (2overlapped $\mathrm{C}=\mathrm{O})$; MS: m/z $293\left(\mathrm{M}+1^{+}, 26.47 \%\right), 291\left(\mathrm{M}-1^{+}, 2.17 \%\right), 264(100 \%)$; Anal. for $\mathrm{C}_{10} \mathrm{H}_{5} \mathrm{BrN}_{4} \mathrm{O}_{2}$ Calcd. (Found): C, 40.98(41.10); H, 1.71(1.75); N, 19.11(18.97).

\section{3-Amino-2-aminocarbonyl-6-bromoquinazolin-4(3H)-one (7b)}

A mixture of 3-Amino-6-bromo-2-ethoxycarbonylquinazolin-4(3H)-one (2b), aqueous ammonium hydroxide solution $33 \%(15 \mathrm{~mL})$ and ethanol $(10 \mathrm{~mL})$ was heated under reflux for $2 \mathrm{~h}$. with continuous stirring. The reaction mixture was then cooled, filtered off and the product was crystallized from ethanol.

Yield: $65.0 \%$; mp: $185-187{ }^{\circ} \mathrm{C}$; IR $\left(\mathrm{cm}^{-1}\right)$; 3300, $3200\left(\mathrm{NH}_{2}\right), 1680,1630(2$ $\mathrm{C}=\mathrm{O}) ; \mathrm{MS}: \mathrm{m} / \mathrm{z} 283.9\left(\mathrm{M}+2^{+}, 20.38 \%\right), 281.9\left(\mathrm{M}^{+}, 20.53 \%\right), 209.95$ (100\%); Anal. for $\mathrm{C}_{9} \mathrm{H}_{7} \mathrm{BrN}_{4} \mathrm{O}_{2}$ Calcd. (Found): C, 38.16(38.50); H, 2.47(2.70); N, 19.78 (19.70).

\section{8-Substituted-1H-[1,2,4]triazino[6,1-b]quinazoline-2,4,10(3H)-triones 8a\&b}

A solution of the respective 3-amino-2-aminocarbonylquinazolin-4(3H)-one $7 \mathrm{a} \& \mathrm{~b}(0.02 \mathrm{~mol})$ and finely grinded urea $(3.6 \mathrm{~g}, 0.06 \mathrm{~mol})$ was fused in an oil bath at 190 ${ }^{\circ} \mathrm{C}$ for $2 \mathrm{~h}$. the crude product was washed with hot water $(30 \mathrm{~mL})$, dissolved in $\mathrm{NaOH}$ $10 \%(100 \mathrm{~mL})$, treated with charcoal, filtered off and precipitated by acetic acid. The formed yellow solid was filtered, washed with water and recrystallized from glacial acetic acid to yield $7 \mathrm{a} \& \mathrm{~b}$. 
1H-[1,2,4]triazino[6,1-b]quinazoline-2,4,10(3H)-triones $(8 \mathrm{a})$

Yield: $75.0 \%$; mp: $>300{ }^{\circ} \mathrm{C}$; IR $\left(\mathrm{cm}^{-1}\right) ; 3500-3200(\mathrm{NH}), 1700,1660,1650$ $(3 \mathrm{C}=\mathrm{O}) ; \mathrm{MS}: \mathrm{m} / \mathrm{z} 231\left(\mathrm{M}+1^{+}, 15.93 \%\right), 230\left(\mathrm{M}^{+}, 87.91 \%\right), 103(100 \%)$; Anal. for $\mathrm{C}_{10} \mathrm{H}_{6} \mathrm{~N}_{4} \mathrm{O}_{3}$ Calcd. (Found): C, 52.18 (51.66); H, 2.62(2.65); N, 24.33 (24.42).

\section{8-Bromo-1H-[1,2,4]triazino[6,1-b]quinazoline-2,4,10(3H)-triones $(8 \mathrm{~b})$}

Yield: $80.0 \%$; mp: > $300{ }^{\circ} \mathrm{C}$; IR $\left(\mathrm{cm}^{-1}\right)$; 3500-3200 (NH),1700,1660,1650 $(3 \mathrm{C}=\mathrm{O}) ;{ }^{1} \mathrm{H}$ NMR (DMSO-d $\left.\mathrm{d}_{6}\right): 5.5\left(\mathrm{~s}, 1 \mathrm{H}, \mathrm{N}^{1} \mathrm{H}, \mathrm{D}_{2} \mathrm{O}\right.$ exchangeable), 7.80-8.40 (m, 3H, Ar-H), 12.20 (s, $1 \mathrm{H}, \mathrm{N}^{3} \mathrm{H}, \mathrm{D}_{2} \mathrm{O}$ exchangeable); Anal. for $\mathrm{C}_{10} \mathrm{H}_{5} \mathrm{BrN}_{4} \mathrm{O}_{3}$ Calcd. (Found): C, 38.86(39.00); H, 1.63(1.59); N, 18.12(18.06).

\section{2-Aryl-8-substituted-3H-[1,2,4] triazino[6,1-b]quinazoline-4,10(3H)-diones $(9 \mathrm{a}-\mathrm{d})$}

A mixture of the respective 3-amino-2-aminocarbonylquinazolin-4(3H)-ones $7 \mathrm{a} \& \mathrm{~b}$ and appropriate aromatic aldehyde $(0.01 \mathrm{~mol})$ in dry dimethylformamide $(25 \mathrm{~mL})$ containing hydrochloric acid $(0.2 \mathrm{~mL})$ was heated under reflux for $24 \mathrm{~h}$. After cooling, the reaction mixture was poured into ice-cold water $(25 \mathrm{~mL})$. The separated yellow solid was filtered off and crystallized from glacial acetic acid.

\section{2-(4-Chlorophenyl)- 3H-[1,2,4]triazino[6,1-b]quinazoline-4,10(3H)-dione (9a)}

Yield: 75.0\%; mp: > $300{ }^{\circ} \mathrm{C}$; IR $\left(\mathrm{cm}^{-1}\right)$; 3300-3100 (NH),1720,1690 (2 C=O); ${ }^{1} \mathrm{H}$ NMR (DMSO-d ${ }_{6}$ ): 7.60-8.30 (m, 8H, Ar-H), 12.40 (s, 1H, N ${ }^{3} \mathrm{H}, \mathrm{D}_{2} \mathrm{O}$ exchangeable); Anal. for $\mathrm{C}_{16} \mathrm{H}_{9} \mathrm{ClN}_{4} \mathrm{O}_{2}$ Calcd. (Found): C, 59.18 (59.20); H, 2.79 (2.90); N, 17.25(17.21).

\section{2-(2-Bromophenyl)- 3H-[1,2,4]triazino[6,1-b]quinazoline-4,10(3H)-dione $(9 \mathrm{~b})$}

Yield: 70.0\%; mp: > $300{ }^{\circ} \mathrm{C}$; IR $\left(\mathrm{cm}^{-1}\right)$; 3300-3100 (NH), $1700,1680(2 \mathrm{C}=\mathrm{O})$; MS: $\mathrm{m} / \mathrm{z} 370\left(\mathrm{M}+2^{+}, 0.09 \%\right), 368\left(\mathrm{M}^{+}, 0.2 \%\right), 228(100 \%)$; Anal. for $\mathrm{C}_{16} \mathrm{H}_{9} \mathrm{BrN}_{4} \mathrm{O}_{2}$ Calcd. (Found): C, 52.05(51.98); H, 2.45(2.64); N, 15.17(15.52).

\section{8-Bromo-2-(4-nitrophenyl)-3H-[1,2,4]triazino[6,1-b]quinazoline-4,10(3H)-dione} (9c)

Yield: $65.0 \%$; mp: $252-254{ }^{\circ} \mathrm{C}$; IR $\left(\mathrm{cm}^{-1}\right)$; 3300-3100 (NH), 1700, $1660(2 \mathrm{C}=\mathrm{O})$; Anal. for $\mathrm{C}_{16} \mathrm{H}_{8} \mathrm{BrN}_{5} \mathrm{O}_{4}$ Calcd. (Found): C, 46.39(46.33); H, 1.94(2.39); N, $16.90(16.83)$.

\section{2-(2-nitrophenyl)-3H-[1,2,4]triazino[6,1-b]quinazoline-4,10(3H)-dione (9d)}

Yield: 60.0\%; mp: $272-274{ }^{\circ} \mathrm{C}$; IR $\left(\mathrm{cm}^{-1}\right)$; 3300-3200 (NH), 1700,1660 (2 C=O); Anal. for $\mathrm{C}_{16} \mathrm{H}_{9} \mathrm{~N}_{5} \mathrm{O}_{4}$ Calcd. (Found): C, 57.31(57.49); H, 2.70(3.11); N, 20.88(20.53).

\section{2-(Trifluoromethyl)-3H-[1,2,4]triazino[6,1-b]quinazoline-4,10-dione (10)}

A suspension of 3-amino-2-aminocarbonylquinazolin-4(3H)-one (7a) (2.04 gm, $0.01 \mathrm{~mol})$ in trifluoroacetic acid $(15 \mathrm{~mL})$ was heated under reflux on a water bath at temperature $38{ }^{\circ} \mathrm{C}$ for $24 \mathrm{~h}$. After cooling, the reaction mixture was poured portionwise carefully onto crushed ice $(30 \mathrm{~g})$. The formed white precipitate was filtered off and crystallized from glacial acetic acid.

Yield: $60.0 \%$; mp: $>300{ }^{\circ} \mathrm{C}$; IR $\left(\mathrm{cm}^{-1}\right) ; 3200(\mathrm{NH}), 1720,1660(2 \mathrm{C}=\mathrm{O})$; MS: $\mathrm{m} / \mathrm{z} 281\left(\mathrm{M}-1^{+}, 2.71 \%\right), 119(100 \%)$; Anal. for $\mathrm{C}_{11} \mathrm{H}_{5} \mathrm{~F}_{3} \mathrm{~N}_{4} \mathrm{O}_{2}$ Calcd.(Found): $\mathrm{C}$, 46.82(46.70); H, 1.78(1.90); N, 19.85(20.00). 


\section{2-(Chloromethyl)-3H-[1,2,4]triazino[6,1-b]quinazoline-4,10-dione (11)}

Chloroacetyl chloride $(1.13 \mathrm{~mL}, 0.01 \mathrm{~mol})$ was added dropwise to a suspension of 3-amino-2-aminocarbonylquinazolin-4 $(3 \mathrm{H})$-one $(7 \mathrm{a})(2,3 \mathrm{~g}, 0.01 \mathrm{~mol})$ in acetic acid $(50 \mathrm{~mL})$. The reaction mixture was heated under reflux for $6 \mathrm{~h}$. and then allowed to cool in an ice bath. The separated solid was filtered off and crystallized from ethanol.

Yield: 61.0\%; mp: > $300{ }^{\circ} \mathrm{C}$; IR $\left(\mathrm{cm}^{-1}\right)$; 3300-3100 (NH), 2960 (C-H aliphatic) $1720,1660(2 \mathrm{C}=\mathrm{O}) ; \mathrm{MS}: \mathrm{m} / \mathrm{z} 264\left(\mathrm{M}+2^{+}, 35.33 \%\right), 263\left(\mathrm{M}+1^{+}, 15.8 \%\right), 262\left(\mathrm{M}^{+}\right.$, $100 \%$ ); Anal. for $\mathrm{C}_{11} \mathrm{H}_{7} \mathrm{ClN}_{4} \mathrm{O}_{2}$ Calcd. (Found): C, 50.30 (49.98); H, 2.68 (2.91); N, 21.33 (21.12).

\section{Antimicrobial activity testing:}

Six of the newly synthesized compounds were evaluated for their in-vitro antimicrobial activity against Staphylococcus aureus (Gram-positive), Bacillus subtilis (Gram-positive), Escherichia coli (Gram-negative), Pseudomonas aeruginosa (Gramnegative) and Candida albicans (Fungi).

\section{Methodolgy}

Using the agar plate disc-diffusion methodology ();agar plates containing $15 \mathrm{~mL}$ of agar medium were seeded with $0.2 \mathrm{~mL}$ of 18 hours broth culture of each organism.

Sterile filter paper discs (6 mm in diameter) were impregnated each with $10 \mu \mathrm{L}$ of a saturated solution of the tested compounds in DMF and allowed to air drying. The discs were then placed onto the surface of agar plates and incubated at $37{ }^{\circ} \mathrm{C}$ for 48 hours.

Control discs impregnated with DMF were used to determine the solvent activity. The diameter of the inhibition zone around each disc was measured in $\mathrm{mm}$.

The bacterial ciprofloxacin reference disc was tested as standard.

\section{Conclusion}

The obtained data (table 1) revealed that compounds $4 \mathrm{~b} \& 5 \mathrm{~b}$ showed mild antibacterial activity against Gram-negative while compounds $8 \mathrm{~b} \& 9 \mathrm{~d}$ showed mild antibacterial activity against Gram- positive, meanwhile compound $4 \mathrm{~b}$ showed also mild antifungal activity.

\section{Analgesic activity testing}

Two comounds were tested for their analgesic activity via " acetic acid Induced Writhing Test' using aspirin as standard.

Methodolgy:

1- Animals:

Adult albino Swiss-Webster mic (18-25 g) of both sex, the animals were divided into four griups each of ten animals. They were housed in a quiet, temperature and humidity-controlled room $\left(22 \pm 3{ }^{\circ} \mathrm{C}\right.$ and $60 \pm 5 \%$ respectivitely).

2- Drug administration:

The synthesized compounds $(15 \mathrm{mg} / \mathrm{Kg})$ and the tested used reference analgesic , aspirin $(15 \mathrm{mg} / \mathrm{Kg})$ were suspended in tween 80 and injected intraperitoneally. 
3- Acetic Acid Induced Writhing Test():

The animals react with characteristic stretching behavior which is called wriyhing, when ininjected with an irritant such as acetic acid $(0.6 \% .10 \mathrm{~mL} / \mathrm{Kg})$ into the peritoneal cavity of the mice.

The first two animal groups were administered the tested compounds in dose (15 $\mathrm{mg} / \mathrm{Kg}$ ) intraperitoneally followed by the intraperitoneal injection of acetic acid $30 \mathrm{~min}$. later. The third group was dosed with the standard reference drug (aspirin) in the same dose of the tested compounds $30 \mathrm{~min}$. before the injection of theacetic acid. Finally the fourth group was injected with tween 80 only as a test control.

The tested mice were observed for the total number of writhines within 20 min. after the acetic acid injection.

The mean values of each group and the percent of reduction of the writhes number were also calculated with respect to these of the control group.

\section{Conclusion:}

The tested compounds $4 \mathrm{~g} \& 9 \mathrm{c}$ were found to decrease the number of writhes induced by the injected acetic acid.

The analgesic activity of the two tested compounds were shown to be more potent than the analgesic activity of asirin. 
Scheme 1:<smiles>[R]n1cnn2c(=O)c3cc(Br)ccc3nc2c1=O</smiles><smiles>CCOC(=O)c1nc2ccc(Br)cc2c(=O)n1/N=C/N</smiles><smiles>CCOC(=O)c1nc2ccc(Br)cc2c(=O)n1N</smiles>
3

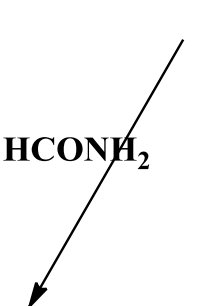<smiles>O=c1[nH]cnn2c(=O)c3cc(Br)ccc3nc12</smiles>

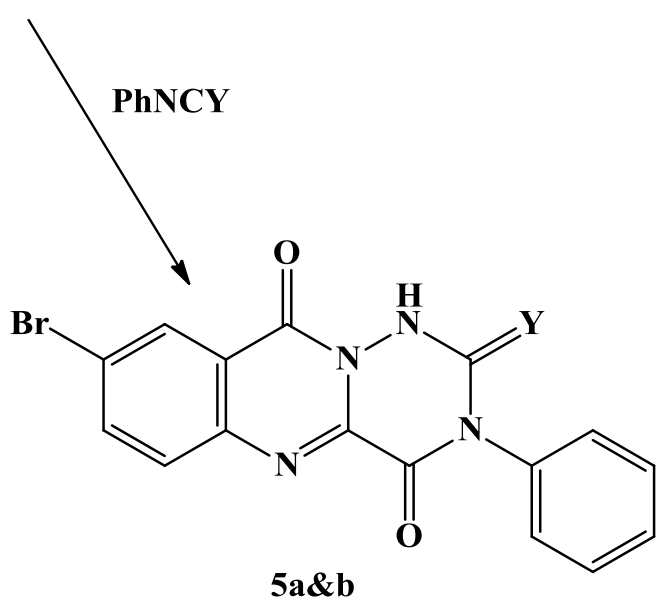

$\mathrm{R}=-\mathrm{CH}_{3},-\mathrm{CH}\left(\mathrm{CH}_{3}\right)_{2},-\mathrm{CH}_{2} \mathrm{CH}\left(\mathrm{CH}_{3}\right)_{2}$, $\mathbf{Y}=\mathbf{O}, \mathbf{S}$ 


\section{Scheme 2:}<smiles>[X]c1ccc2nc(C(=O)OCC)n(N)c(=O)c2c1</smiles>

$\mathbf{2 a \& b}$<smiles>[X]c1ccc2nc(C(N)=O)n(N)c(=O)c2c1</smiles>

$7 \mathbf{a} \& \mathbf{b}$

$\mathbf{X}=\mathbf{H}, \mathbf{B r}$<smiles>Cc1ccc([N+](=O)[O-])cc1</smiles><smiles>[X]c1ccc2nc3c(=O)[nH]c(=O)[nH]n3c(=O)c2c1</smiles>

ArCHO<smiles></smiles><smiles>O=c1[nH]c(C(F)(F)F)nn2c(=O)c3ccccc3nc12</smiles>

\section{$\mathrm{ClCOCH}_{2} \mathrm{Cl}$}<smiles>O=c1[nH]c(CCl)nn2c(=O)c3ccccc3nc12</smiles> 
Table 1: Antimicrobial activity of newly synthesized compounds on different microorganisms.

\begin{tabular}{|l|l|l|l|c|c|}
\hline \multirow{4}{*}{ Compound no. } & \multicolumn{5}{l|}{ Diameter of zone of inhibition in mm } \\
\cline { 2 - 6 } & \multicolumn{5}{|l}{ In vitro activity against } \\
\cline { 2 - 6 } & S. aureus & B. subtitlis & E. coli & P. aeruginosa & C. albicans \\
\hline 4b & - & - & 5 & - & 5.5 \\
\hline 5b & - & - & - & 5 & - \\
\hline 8b & 6 & - & - & - & - \\
\hline 9d & 6 & - & - & - & - \\
\hline $\mathbf{1 0}$ & - & - & - & - & - \\
\hline $\mathbf{1 1}$ & - & - & - & - & - \\
\hline DMF & - & - & - & 11 & - \\
\hline Ciprofloxacin & 9 & 10 & 10 & & - \\
\hline
\end{tabular}

Table 2: Analgesic activity of newly synthesized compounds

\begin{tabular}{|l|c|c|c|}
\hline Compound no. & $\begin{array}{c}\text { Mean of the no. of } \\
\text { writhes within 20 } \\
\text { min. }(\mathbf{x})\end{array}$ & $\begin{array}{c}\text { Standard Error } \\
\text { (SE) }\end{array}$ & $\begin{array}{c}\text { \% Reduction from } \\
\text { the control } \\
(\mathbf{x}-\mathbf{c} / \mathbf{c}) \times \mathbf{1 0 0}\end{array}$ \\
\hline $\mathbf{4 g}$ & 1.667 & 0.527 & 62.95 \\
\hline $\mathbf{9 c}$ & 0.80 & 0.132 & 82.20 \\
\hline Aspirin & 2.1 & 0.314 & 53.33 \\
\hline Control & 4.5 & 0.289 & - \\
\hline
\end{tabular}

\section{REFERENCES}

Abdel-Hamide S.G.,(1997): J. Ind. Chem. Soc., 74, 613.

Abdel-Hamide S.G.,(2001): Saudi Pharmaceutical Journal, 9(2), 72.

Abdel-Mageed, M.F., Teniou, A., (1988): Collect. Czech. Chim. Commun., 53(2), 329.

Alafeefy A.M., Kadi A.A., Al-Deeb O.A., El-Tahir K.E., Al-Jaber N.A., (2010): Eur. J. Med. Chem., 11, 4947.

Alagarsamy V., Solomon V.R., Dhanabal K., (2007); Bioorg. Med. Chem., 15, 235.

Bansal E., Ram T., Sharma, S., Tyagi M., Rani A.P., Bajaj K., Taygi R., Goel B., Srivastava V.K., Guru, J.N., Kumar, A., (2001): Ind. J., Chem., 40B(4), 307. 
Chandrika P. M., Yakaiah T., Rao A.R.R., Narsaiah B., Reddy N. C., Sridhar V., Rao J.V., (2008): Eur. J. Med. Chem., 43, 846.

Casgrande, C., Canova M., Ferrari G., (1965): Farmaco, 20(8), 544.

Elsilk S.E., El-barbary A.A., 2010: Middle-East J. of Sci. res. 6(1), 31.

George T., Mehta D.V., Tahilramani R., (1971): Ind. J., Chem., 9, 755.

Giri R.S., Thaker H.M., Giordano T., Williams J., Rogers D., Sudersanam V., Vasu K. K., (2009): Eur. J. Med. Chem., 44, 2184.

Ghorab M.M., Ismail Z.H., Abdalla M., Radwan A.A., (2013): Arch. Pharm. Res., 36,660 .

Gucky’ T., Reznickova E., Dzubak P., Hajduch M., Krystof V., (2010): Monatsh Chem., 141, 709.

Hussein M.A., (2012): Med Chem Res, 21, 1876.

Kovalenko S.I., Nosulenko I.S., Voskoboynik A.Y., Berest G.G., Antipenko L.N., Antipenko A.N., Katsev A.M., (2013): Med. Chem. Res., 22, 2610.

Minu M., Thangadurai A., Wakode S.R., Agrawal S.S., Narasimhan B., (2008):

Arch. Pharm. Chem. Life Sci., 341, 231.

Mulakayala N., Kandagatla B., Ismail, Rapolu R.K., Rao P., Mulakayala C., Kumar C.S., Iqbal J., Oruganti S., (2012): Bioorg. Med. Chem. Letters, 22, 5063.

Noolvi M.N., Patel H.M., Bhardwaj V., Chauhan A., (2011): Eur. J. Med. Chem., 46, 2327.

Panneerselvam P., Rather B.A., Reddy D.R.S., Kumar N.R., (2009): Eur. J. Med. Chem., 44, 2328.

Pathak, U.S., Rathod I.S., Patel M.B., Shirsath V.S., Jain K.S., (1995): Ind. J., Chem., 44B, 617.

Ryu C.K., Kim Y.H., Im H.A., J.Y. Kim, Yoon J.H., Kim A., (2012): Bioorg. Med. Chem., 22, 500.

Taha M.A.M., (2007): Monatshefte fur Chemie 138, 505. 


\section{الملخص العربي \\ تثييد بعض مركبات ترايزينوكينازولين جديدة ذات تأثير بيولوجى متوقع

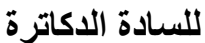

مرفت مصطفى العنانى، عفاف كمال الدين الانصارى، منى محمد عبد الجو اد، ايهاب محمد عبدالمنعم جداوى

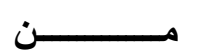

$$
\text { قسم الكيمياء العضوية الصيدلية ـ كلية الصيدلة - جامعة القاهرة - مصر }
$$

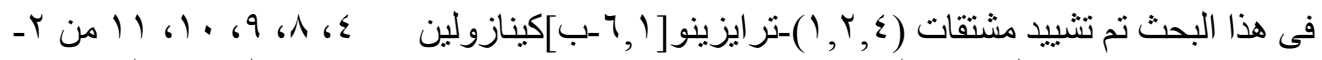

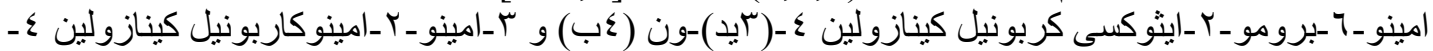

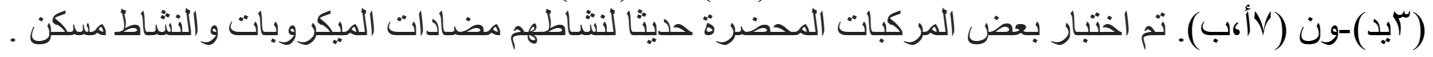

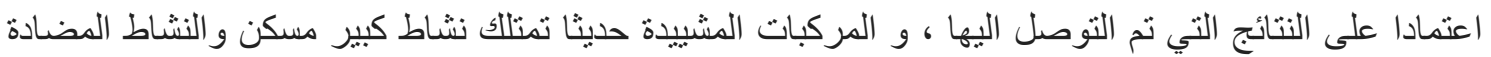
للميكروبات خفيفة التئة 